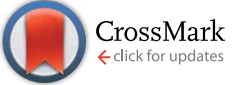

Cite this: RSC Adv., 2017, 7, 8443
Received 9th November 2016 Accepted 5th January 2017

DOI: 10.1039/c6ra26526j

www.rsc.org/advances

\section{Rapid fabrication of robust, washable, self-healing superhydrophobic fabrics with non-iridescent structural color by facile spray coating $\dagger$}

\author{
Qi Zeng, ${ }^{a}$ Chen Ding, ${ }^{a}$ Qingsong Li, ${ }^{a}$ Wei Yuan, ${ }^{b}$ Yu Peng, ${ }^{a}$ Jianchen $\mathrm{Hu}^{\star a}$ \\ and Ke-Qin Zhang*a
}

\begin{abstract}
Robust, washable, and self-healing superhydrophobic fabrics with non-iridescence structural colors were prepared by a one-step spray coating technique using easily available materials: polyacrylate (PA) adhesive, monodisperse poly(styrene-methyl methacrylate-acrylic acid) (P(St-MMA-AA)) colloidal microspheres, and carbon black (CB). The color of the coated fabrics could be tuned as violet, green or red by just varying the size of the microspheres, and this method was effective in enhancing the fastness of the structural color on fabrics; for example, the coated fabrics could repeatedly withstand standard laundry, expedite laundry, and even supersonic vibrating cleaning without apparent color fading. In addition, the as-formed fabrics maintained superhydrophobicity with a high contact angle $\left(>150^{\circ}\right)$. The coated fabrics could also restore its super liquid-repellent property by a short-time heating treatment or room temperature ageing after being tentatively damaged. This simple but effective coating method may facilitate the development of special functional clothing for various applications.
\end{abstract}

\section{Introduction}

Vivid structural color can often be seen in nature, which is fadeless and dye-free in contrast to the traditional dyes or pigments. It mostly arises from interference, diffraction, or scattering of light from micro- or nanostructures with the scale on the order of light wavelength. ${ }^{1-3}$ These natural structures producing special color provide great sources of inspiration in biomimetic technology for the design or fabrication of novel color-related optical materials and devices. ${ }^{4}$ This new dyeing technology has attracted significant attention in a wide variety of research fields ${ }^{5}$ as it may avoid severe water pollution introduced by chemical dyes or synthetic pigments. As representative, conventional photonic crystals (PCs) with both short- and long-range order arrangements produce iridescent structural colors such as the colors of beautiful butterfly wings, beetle scales, and opals generated due to the photonic bandgap effect. $^{6-9}$ However, the structural colors produced by colloidal crystals show distinct variations depending on the viewing and light illumination angles. ${ }^{\mathbf{1 0}, 11}$ This iridescence confines the real application of colloidal crystals as pigments because for typical

${ }^{a}$ National Engineering Laboratory for Modern Silk, College of Textile and Clothing Engineering, Soochow University, Suzhou 215123, China. E-mail: kqzhang@suda. edu.cn

${ }^{b}$ Suzhou Institute Nano-Tech and Nano-Bionics (SINANO), Printed Electronics Division, Chinese Academy of Sciences, Suzhou 215123, China

$\dagger$ Electronic supplementary information (ESI) available. See DOI: $10.1039 /$ c6ra26526j pigments, constant colors are generally required even at different viewing angles. Iridescences originate from Bragg reflection as a result of the long-range order in the particle arrangement; ${ }^{\mathbf{1 2 - 1 4}}$ thus, the direct solution for the suppression of iridescences would be breaking down the crystalline structure arrangement of PCs to establish an amorphous state. Recently, amorphous photonic structures (APS) have attracted significant attention as their unique structural features of only short-range order result in many interesting optical properties, such as unusual light scattering, transport, and localization. ${ }^{\mathbf{1 5}}$ Moreover, APSs can produce vivid non-iridescent structural colors, such as the color of the birds Cotinga maynana and L. coronata, the blue skin of mandrills or the scales of longhorn beetles, when the structural feature sizes are comparable to the wavelength of visible light. The non-iridescence structural colors of APSs originate from an isotropic photonic pseudo gap $^{\mathbf{1 6 - 2 0}}$ as a result of their short-range order arrangement. Due to the broad viewing angles, these non-iridescent structural colors have important potential applications in color-related fields such as paints, cosmetics, textile, or displays. ${ }^{6}$ Currently, self-assembly methods, including spin coating, drop coating, spray coating, and so on, have been mostly utilized to obtain APSs. ${ }^{15}$ All these methods can suppress the long-distance electrostatic interactions among spheres and break down the long-range order arrangement of the colloidal structures to reduce the iridescent phenomenon. Among these techniques, spray coating has the obvious benefit of rapid patterning over a large area, and the quick self-assembly contributes to the formation of APSs. ${ }^{11,21,22}$ 
A number of studies have focused on creating structural iridescent color or non-iridescent color, but one problem that can't be completely solved is that the color may become weak once liquids infiltrate into the gap of the structural matrix, leading to a decreased refractive index contrast for the two mediums in the structural matrix. ${ }^{23-25}$ This shortage dramatically limits the application of structural color materials, especially in textiles. It has been observed that many living organisms are able to retain brilliant colors even under wet conditions because of the extra transparent and waterproof coating or rough array on their colored surface. This enables a self-healing function by regenerating the protection, ${ }^{21,26,27}$ which is present on the wings of the morpho butterfly, the peacock feather, and beetle shells, against chemical or physical damages. To mimic the hydrophobic non-iridescent structural colors in nature, some groups used hydrophobic nanoparticle pigments to prepare superhydrophobic, angle-independent colors. $^{28}$ Ge's group created superhydrophobic, angleindependent colored films by one-step spray coating of monodispersed, fluorosilane-functionalized silica NPs suspended in isopropanol. ${ }^{21}$ Wang and his colleagues created superhydrophobic colored films by spray coating poly(styrene- $n$-butyl acrylate-acrylic acid) core-shell particles. ${ }^{22}$ However, fluoride materials are not environmentally friendly, and the fastness of color and stability of the superhydrophobicity are also big challenges, as well as the self-healing function. Wu and coworkers recently considered that using anisotropic Ag nanoparticles (AgNPs) as non-toxic and eco-friendly dyes, like many researchers, ${ }^{28-35}$ and then depositing the AgNPs with tunable surface plasmon resonance would endow the fabrics with abundant color. The major obstacles were the poor color fastness and the fabrication difficulty. They reported a three-step method for the fabrication of poly(ethylenimine)/AgNPs/ poly(ethylenimine)-coated cotton fabrics with tunable colors as well as durable antibacterial and self-healing superhydrophobic properties by a sequential solution-dipping process. The color fastness and durability of the silvernanoparticle-coated fabrics were improved, but not the color saturation and fabric handling. ${ }^{36}$ Many fluorine-free fabrics with robust superhydrophobicity have been successfully fabricated by introducing hydrophobic Si nanoparticles and longchain hydrophobic alkyls on cotton fabrics, ${ }^{37}$ whereas tiny and heterogeneous $\mathrm{SiO}_{2}$ NPs could not produce any other color than white. Poly(styrene-methacrylic acid) (P(St-MMA)) photonic crystal $^{38-40}$ is also a popular candidate for the fabrication of hydrophobic textile fabrics with structural colors; however, brilliant non-iridescent structural color fabrics based on it are rarely investigated. Moreover, from the functionalization point of view, systematic evaluation of the fabric properties, such as washability, fastness, and hydrophobicity, is essential for both scientific research and industry. ${ }^{41}$

In this study, we have fabricated an APS dyeing system by a facile spray coating technique and the produced noniridescent color is tunable in the visible spectrum. We found that the APSs are strongly related to the diameter of the colloid spheres, whereas their spectral purity can be controlled by the proportion of colloid spheres and carbon black (CB), and the color depth can be tuned by spraying times. More importantly, the whole dyeing procedure is rather fast within 20 seconds and a transient heat treatment endows the fabrics to be washable and robust with superhydrophobic properties. It is conceivable that this novel dyeing technique on fabrics based on the structural color will provide a new approach to prepare functional textiles and revolutionize the fashion industries.

\section{Experimental}

\section{Materials and sample preparation}

All chemicals were of reagent grade and were used without further purification as purchased. Styrene (St), methyl methacrylate (MMA), acrylic acid (AA), ammonium persulfate (APS), sodium dodecyl benzenesulfonate (SDBS), ammonium bicarbonate, and ethanol were purchased from Shanghai Chemical Reagent Co. Ltd (China). Carbon black (CB) with an average particle diameter of $28 \mathrm{~nm}$ was obtained from Tokai Carbon Co. Ltd. Deionized (DI) water was used in all the experiments. Polyacrylate adhesive (PA) and black plain cotton fabrics (160 g $\mathrm{m}^{-2}$, thickness $=520 \mu \mathrm{m}$ ) were bought from the local market and were rinsed with acetone and deionized water and cut into the size of $4 \mathrm{~cm} \times 5 \mathrm{~cm}$ (each sample) before use.

\section{Synthesis of monodispersed P(St-MMA-AA) composite nanospheres}

Monodispersed composite latex spheres of poly(styrene-methyl methacrylate-acrylic acid) (P(St-MMA-AA)) were prepared according to the previously reported method (emulsion polymerization)..$^{42}$ Briefly, St (38 ml), MMA $(2 \mathrm{ml})$, AA ( $\left.2 \mathrm{ml}\right)$, DI water $(200 \mathrm{ml})$, SDBS $(0-0.033 \mathrm{~g})$, and ammonium bicarbonate $(1 \mathrm{~g})$ were sequentially added to a four-necked flask equipped with a $\mathrm{N}_{2}$ inlet, a reflux condenser, and a mechanical stirrer at a stirring speed of $300 \mathrm{rpm}$. The reaction mixture was initially performed at $70{ }^{\circ} \mathrm{C}$ for $30 \mathrm{~min}$. Following the addition of an aqueous solution of APS (1.05 $\mathrm{g}$ dissolved in $2 \mathrm{~g}$ DI water), polymerization was carried out at $80{ }^{\circ} \mathrm{C}$ for $10 \mathrm{~h}$ under continuous stirring. The resulting latex spheres redispersed in DI water were washed 3 times by centrifugation and ultrasonication, maintaining the concentration of $10 \mathrm{wt} \%$.

\section{Preparation of the coating pigment}

The concentration of PA was regulated to $26 \mathrm{wt} \%$ by centrifugation. According to the weight ratio of $100: 2: 1$, P(St-MMAAA) colloidal particulate solution/PA/CB, as a special coating pigment, was systematically prepared to form a homogenous solution. After 20 min of magnetic stirring, the blended solution was ready for spray coating on fabrics. To study the relationship between amount of CB and color saturation, the amount of CB suspended in the solution was varied from 1,2 , to $5 \mathrm{wt} \%$.

\section{Ultra-fast preparation of colourful and functional fabrics}

The as-prepared suspension was added to the airbrushed and sprayed fabric under a pressure of $50 \mathrm{kPa}$. The fabric substrate was sprayed for 5 times, for 10 seconds each time. The time 
interval of spraying was 20 seconds. The treated fabric was then dried at $80{ }^{\circ} \mathrm{C}$ for $1 \mathrm{~h}$ in an oven.

\section{Characterization}

The morphologies of the colloidal spheres, original fabrics, and structural color fabrics before and after washing, rubbing, and plasma treatment were observed by a field emission scanning electron microscope (FE-SEM) (S-4800, Hitachi Ltd, Japan). The samples were sputtered with a gold film before SEM observation. Water contact angles (CAs) and wetting time of the original and coating fabrics were measured using a contact-angle system (Easy Drop, Germany) at ambient temperature and proper humidity. The washing durability was evaluated by reference of national standard washing method (GB/T 3921-2008) and the washing procedure described in the AATCC (American Association of Textile Chemists and Colorists) Test Method 61-2006 test No. 2A, respectively. The test was performed using a standard laundering machine (SW-12A, Wuxi textile machinery Co., Ltd) equipped with a $500 \mathrm{ml}(75 \mathrm{~mm} \times 75 \mathrm{~mm})$ stainless-steel lever-lock canisters. ${ }^{43}$ The coated fabrics were subjected to a vacuum plasma treatment using a purpose made plasma machine consisting of a vacuum chamber, a radio-frequent plasma generator (T \& C Power Conversion, Inc. AG0201HV), an electrode system, and a gas supply system. The $L \times a \times$ $b$ values and $K / S$ value were determined. All the lightness and $K /$ $S$ values were obtained using color measuring \& matching instrument (DATACOLOR 600).

\section{Results and discussion}

The process of structural color fabrication on fabrics is illustrated in Scheme 1a. Paints combining poly(styrene-methyl methacrylate-acrylic acid) (P(St-MMA-AA)) composite nanospheres (NPs), polyacrylate adhesive (PA), and carbon black (CB) were uniformly mixed in an appropriate ratio, followed by

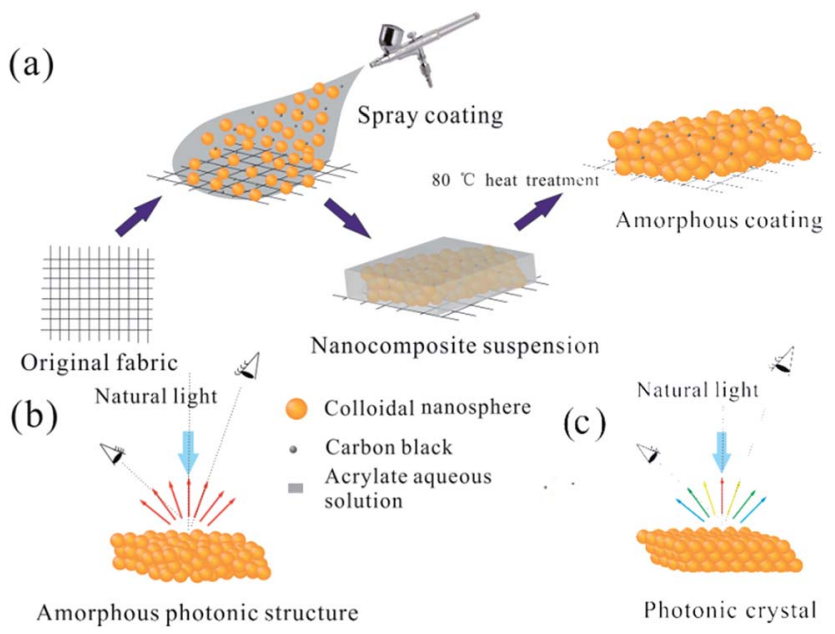

Scheme 1 (a) Schematic for the fabrication procedure of functional fabrics with non-iridescent structural color. (b) Schematic of amorphous photonic structure with non-iridescence. (c) Schematic of photonic crystal structure with iridescence. magnetic stirring for 20 min. The P(St-MMA-AA) composite NPs with a hydrophobic core of poly(styrene) (PS) and a hydrophilic shell of poly ( $n$-methyl methacrylate-acrylic acid) (PMMA-PAA) were prepared as the main element of the coating paint synthesized by emulsion polymerization process. ${ }^{42}$ The special core-shell structure would have a wettability transition under different temperatures. With the incorporation of PA, the massive number of carboxyl groups anchored on the shell of the NPs (Fig. S1†) would improve the adhesion effect between the NPs and fabrics. The introduction of PA aimed to provide excellent washability and stability without a heavy influence on the coated color. The importance of PA has been discussed later. To ensure the coating uniformity and dyeing speed, the blended coating solution was air-sprayed on a black plain cotton fabric approximately $10 \mathrm{~cm}$ from the exit of the $0.2 \mathrm{~mm}$ spraying nozzle. Under a gas pressure of $50 \mathrm{kPa}$, the aqueous solution rapidly volatilized forming quasi-APS with short-range ordering by non-equilibrium assembly, breaking down the ordered arrangement of the NPs. The as-formed fabrics would show angle-independence vivid color within 20 seconds (Scheme 1b; Movie S1, ESI: $\dagger$ the solution was P(St-MMA-AA) composite NPs with diameter of $294 \mathrm{~nm}$ in the concentration of $10 \mathrm{wt} \%$, and the substrate was black plain weave fabric). Then, the colorful fabrics were treated via a transient heating treatment, and the APS was form on the surface of the fabrics. Note that the result was obviously different from the situation that the nozzle and fabrics were too close or the concentration of the solution was too low, by which spraying of the suspension resulted in the formation of a thin liquid layer on the fabric's surface. ${ }^{44,45}$ In these cases, the NPs would easily crystallize to form an ordered photonic crystal structure during the evaporation of the aqueous solution and display iridescent colors (Scheme 1c).

To achieve the expected non-iridescent effect, the first step was forming structural color with the NPs and the color should be tunable. It was observed that the color of the fabrics depended on the size of the used particles when spray parameters were constant. In this study, fabrics were generally treated by spraying them 5 times. As shown in Fig. 1a, the fabrics formed by spraying P(St-MMA-AA) composite NPs with diameters of $217 \mathrm{~nm}, 256 \mathrm{~nm}$, and $294 \mathrm{~nm}$ (Fig. S2 $\dagger$ ) showed brilliant angle-independent violet, green, and red colors, respectively. The corresponding reflective spectra of the colorful fabrics are shown in Fig. $2 b$, exhibiting distinct peaks at approximately $420 \mathrm{~nm}, 507 \mathrm{~nm}$, and $650 \mathrm{~nm}$, which were consistent with the color observed by the naked eyes. Compare to the reflective spectra of the previously reported photonic crystal structures, ${ }^{46-48}$ the overall reflections, as shown in Fig. 1b, were rather low and had a bigger width of the reflection peaks. The peak of reflection spectrum in the visible light region appeared as a result of constructive interference of light. Intensity of the overall reflection was low and the width of the peak was large due to the lack of photonic band in the samples, which originated from the incoherent multiple scattering of light.9 ${ }^{4950}$ Fig. 1c shows the SEM images of the arrangement of the particles on the green fabric, in which the colloid particles appear assembled into an amorphous structure, rather than crystalline 
(a)

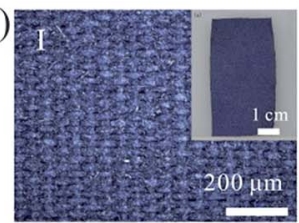

(c)

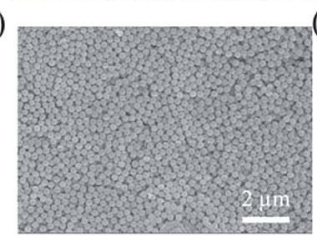

(g)

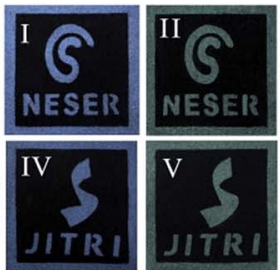

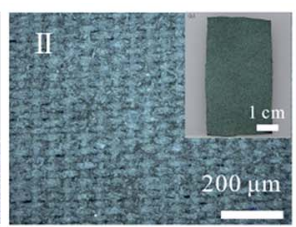

(d)

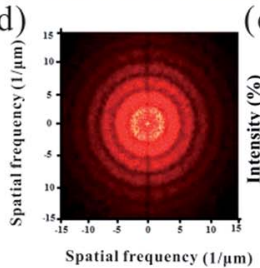

(e)
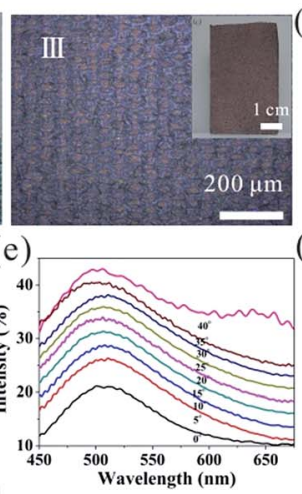

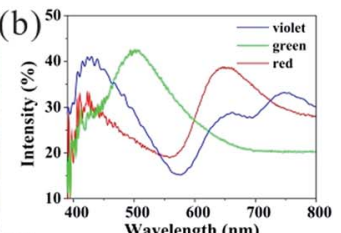

(f)

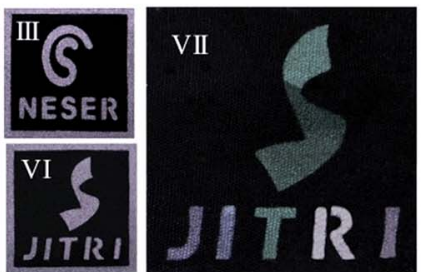

(h)

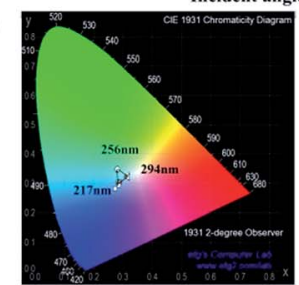

Fig. 1 Optical properties and microstructure of the fabrics with structural color prepared with spray coating technique. (a) Optical images of the colorful fabrics prepared with nanoparticles with diameter of (I) $217 \mathrm{~nm}$ (left), (II) $256 \mathrm{~nm}$ (middle), and (III) $294 \mathrm{~nm}$ (right). (b) Reflection spectra of the coated fabrics, as shown in (a) with particle diameter of $217 \mathrm{~nm}$ (violet), $256 \mathrm{~nm}$ (green), and $294 \mathrm{~nm}$ (red). (c) SEM image of the surface of the coated fabrics. (d) Two-dimensional Fourier power spectra of the SEM image. (e) Reflection spectra of the coated fabric by nanoparticles with diameter of $256 \mathrm{~nm}$, measured at various incident angles. (f) Plots of the positions of the reflection peaks versus the light incident angle. (g) Our lab logo colored by three non-iridescent structural colors (I-VI), and precisely controllable structural colors logo (VII). The fabric size was $3 \mathrm{~cm} \times$ $3 \mathrm{~cm}$. (h) CIE chromaticity $x$ and $y$ values for the colors in the palette.

(a)

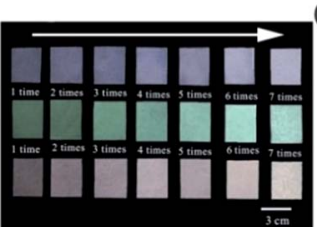

(c) 100

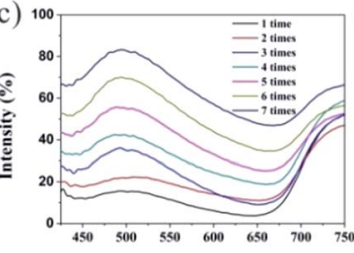

(e)

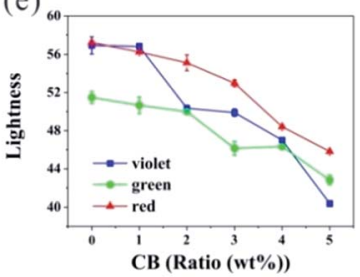

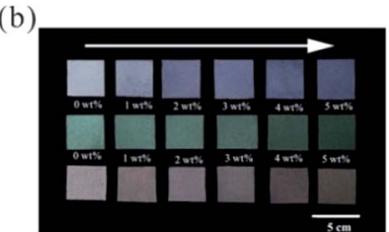

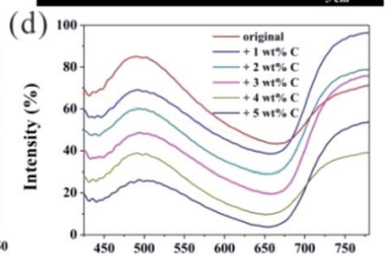

(f)

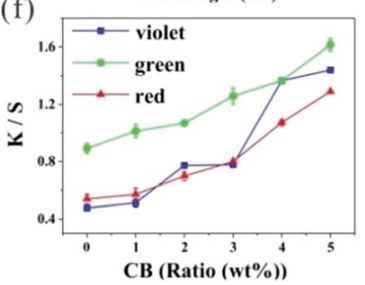

Fig. 2 (a) Optical images of the fabrics coated with NPs with different diameters, and the coating thickness is controlled by the spray time ranging from 1 to 7 (left to right). (b) Optical images of the fabrics coated with NPs/CB mixtures, and the ratio of CB in each sample (left to right) is $0,1,2,3,4$, and $5 \mathrm{wt} \%$, respectively. (c) Reflection spectra of the coated fabrics prepared with $256 \mathrm{~nm}$-sized NPs in different spray times. (d) Reflection spectra for the coated fabrics prepared with 256 $\mathrm{nm}$-sized NPs mixing with different amounts of CB after spraying 5 times. (e) Lightness of the 3 coated fabrics plotted against the amount of $C B$. (f) Plots of $K / S$ value of the 3 coated fabrics against the amount of $\mathrm{CB}$.

structure. Two-dimensional Fourier analyses of the morphology of the coated fabric, as shown in Fig. 1d, revealed that it had a disc pattern with an intense edge, indicating that the structures on the fabrics were essentially isotropic and had a well-defined short-range order. ${ }^{51}$ To prove the formation of an angleindependent color of the coated fabrics, the angle-resolved specular reflection of the green-coating fabrics was measured, as shown in Fig. 1e. The reflection peak of the spectra barely shifted when the observing position was moved from $0^{\circ}$ to $40^{\circ}$, corresponding to the same peak position observed in the reflection spectrum (Fig. 1f). Thus, the structure on the surface of the fabrics had general characteristics of APS: coherent scattering due to the short-range order in the structure led to a photonic pseudogap, and photonic density of the corresponding structure was non-zero, which made the color of the fabrics much softer that met people's need. This distinct controllable and non-iridescent property of APSs is important for some applications such as the microscopic color display. As a demonstration of the controllable coloring effect, these artificial structural colors produced by spray coating were used to manifest the logo of our lab, as shown in Fig. 1g. Three colors, violet, green, and red were obvious, and patterning can be precisely controlled, as seen in Fig. $1 \mathrm{~h}$. The coating fabrics' reflective peaks in the spectra were converted to Commission Internationale de L'Eclairage (CIE) Tristimulus values, the corresponding chromaticity diagram. It almost covered the entire visible spectrum using nanoparticles of sizes $217 \mathrm{~nm}, 256 \mathrm{~nm}$, and $294 \mathrm{~nm}$. 
Except for hue, the color lightness and saturation are also important in real applications. In this study, the lightness of the coated fabrics was tunable by the coating thickness, which could be controlled by changing the spray times. Generally, the color became whiter and brighter from distinct and deep along with the increasing thickness. To analyze the color of the fabrics dyed at different spray times, 7 coated fabrics (with average particle diameters, Dave, of $217 \mathrm{~nm}, 256 \mathrm{~nm}$, and $296 \mathrm{~nm}$ ) with different spraying times were prepared. As listed in Fig. 2a, the coating fabrics were obtained by varying the spray times from 1 (left) to 7 (right), and the color of these gradually became less saturated and whitey with the increasing spray time. The cut samples were located on a rough black substrate for obtaining optical images to eliminate the disturbance of the reflected light from the background. The particles were attached on the surface of each fiber and gradually covered its original coating, as shown in Fig. S3. $\dagger$ Thus, the enhancement of coherent-light scattering improved the lightness of the fabrics, but fabrics exhibited a whitish color. Importantly, the colors were still maintained even when observed from oblique directions, which basically satisfied people's requirements on color effect of textile. Fig. 2c shows the corresponding reflection spectra of the green samples with different spraying times, and the reflection spectra of other samples are shown in Fig. S4.† Reflection spectra of the fabrics after spraying just once barely showed an obvious peak, and the color of the fabrics was inconspicuous with a reflection intensity of less than $15 \%$. After spraying three times, a small peak appeared. On repeating the spraying process 7 more times, the intensity of the reflection peak gradually enhanced from $36 \%$ to $84 \%$. Eventually, the spectra had an obvious peak in the green light region, but a shortage in the intensity was concomitant. When the reflection intensity was beyond $40 \%$, a bright and whitish color could be observed by naked eyes. This was because although the APS on the fabrics could reveal the wavelength-specific constructive interference of light, the observation of structural color from the thicker array was inconspicuous for the large contribution from the incoherent multiple scattering of light. ${ }^{52-54}$

It was observed that the color saturation could be controlled by incorporating small amounts of black particles, which could uniformly absorb light covering the entire visible region. ${ }^{55} \mathrm{CB}$ is one of the most common and environmentally friendly black substances and reflects very little light in the visible region. ${ }^{56}$ We fabricated fabrics with different color saturation by varying the amount of CB particles with an average size of $28 \mathrm{~nm}$, and all the fabrics were still sprayed 5 times. The different hue of the fabrics prepared with NPs/CB mixtures in the same procedure are listed in Fig. 2b. The color became more saturated as the amount of $\mathrm{CB}$ was increased from 0 to $5 \mathrm{wt} \%$, and the color would be dim and vague if the concentration of CB went beyond $5 \mathrm{wt} \%$. Fig. 2d-f quantitatively show the reflectance spectra, lightness, and $K / S$ value (dyeing depth, absorption coefficient $K$, and scattering coefficient $S$ ) of the green coated fabrics with different ratios of $\mathrm{CB}$, and variation diagram of color saturation of the other two can be found in Fig. S5. $\dagger$ The reflection intensity decreased from $86 \%$ to $27 \%$ as the incorporation of CB increased from 0 to $5 \mathrm{wt} \%$, whereas the ratio of the magnitude of the peak component to the total reflectance and position of the peak components nearly stayed constant. The incorporated CB absorbed more of the light in the structure over the entire spectral range of visible light. Due to the enhancement of the structural color saturation by reducing the incoherent wavelength-independent scattering of light across the entire visible region, structural colors from coated fabrics could be clearly observed. ${ }^{57}$ However, as seen in Fig. 2e and f, lightness of the coating fabrics also decreased with the incorporation of more CB. On the contrary, the color depth of the fabrics had a tendency to increase as the amount of CB increased. This rule may enable us to find the right way to fabricate fabrics with structural colors satisfying peoples' needs by facilely regulating appropriate spraying times and the amount of CB.

Except for the coloring effect, comfort is another important concern for fabrics. Basically, the comfort of fabrics depends on the material properties and the weave style. To maintain the comfort of the fabrics, the coating layer should follow the fluctuation of the fibers after introducing structural color NPs. At least the NPs' assembly should match the microstructure on the surface of the fabrics to maintain the flexibility of the fabrics. The obtained fabrics with structural color fabricated by the spraying method are shown in Fig. 3. The surface morphology of fabric didn't apparently change after spray coating (panel I). The surface of the coated fabrics showed a thin membrane compared with the original ones (panel II), and most of the NPs covered on the fibers or even permeated into the gap among the fibers. Fortunately, the warps and weft yarns on the surface of the coating fabrics could still be clearly observed, even every fiber in the yarn. PA was added as a water soluble adhesive with high viscosity. Before solidification, PA filled in the gap among the NPs and fibers. Assembled NPs were firmly fixed on the surface of each fiber after temperature

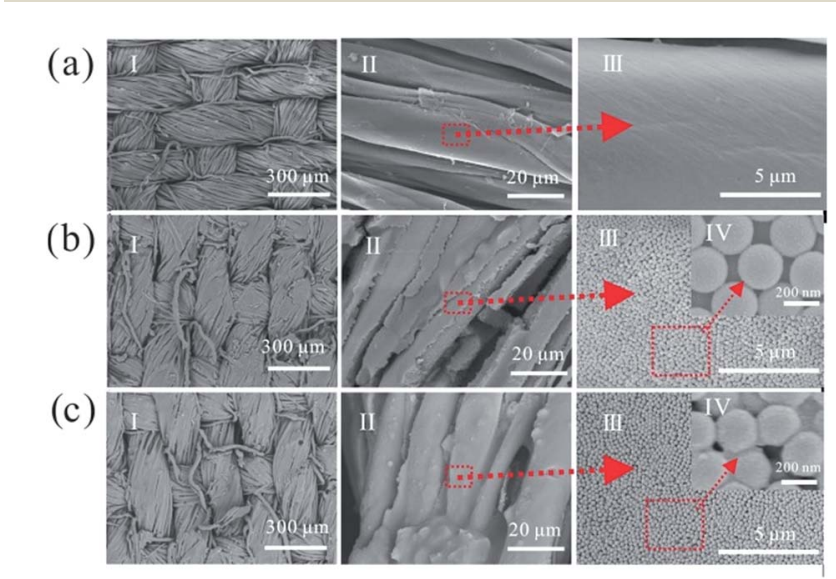

Fig. 3 (a) SEM image of the original fabric, (al and all) shows the morphology of the fabric and fiber, and magnified view of the redframed region is shown in (allI). (b) SEM image of the coated fabric without PA, (bl and II) shows quasi-amorphous ordering structure on the surface of the fabrics observed from the magnified image shown in (bIII), and inset of (bIII) are the nanoparticles with clear boundary. (c) SEM images of coated fabric with PA, in which (clll) is the magnified view of ( $\mathrm{Cl}$ and II) showing quasi-amorphous ordering, and inset of the SEM image in (cIII) shows the nanoparticles adhered to each other. 
treatment. Effect of the incorporation of PA on the fabrics' comfort was also studied. As shown in Fig. 3b and c, the incorporation of PA basically had no impact on the morphology of the fabrics. Fig. 3cIV shows that PA covered the surface to connect separate NPs. PA provided the adhesive force between the colloidal spheres, whereas the surface of NPs without PA (Fig. 3bIV) was very smooth and there was an obvious edge between the colloidal spheres. Therefore, incorporation of PA provided a stronger bonding ability without heavy breaking of the fabric morphology.

To testify the binding effect of the introduced PA, washing and abrasion durability of the coating fabrics were tested. We simulated ordinary home laundering under the condition of $40{ }^{\circ} \mathrm{C}$ as washing temperature in the standard soap bath $(5 \mathrm{~g}$ $\left.\mathrm{L}^{-1}\right)$, and the ratio of fabric and soap solution was $1: 50(\mathrm{w} / \mathrm{w})$. After 10 cycles of the standard machine laundry, the coated fabrics still maintained its vivid color (Fig. 4a). The inset image shows a comparison of the original fabric and coated fabric washed for 10 times, in which the color of the compared samples didn't show apparent distinction. Fig. 4b shows the corresponding reflection spectra of the coated fabrics by standard machine laundry for different cycle times, and the intensity and peak wavelength of each coated fabric sample in the spectra almost remained the same. Fig. 4c shows that the lightness and $K / S$ value of the coated fabrics always remained at the same level during the whole process of washing. Test of harder washing condition on the coated fabrics according to American Association of Textile Chemists and Colourists (AATCCA 61-2006) standard method under 2A condition was also carried out. As shown in Fig. 4d, color of the coated fabrics nearly unchanged after expedite machine laundry from one to 10 times. Moreover, under abrasion of dozens of small steel balls, $49{ }^{\circ} \mathrm{C}$ washing temperature and $150 \mathrm{ml}$ aqueous solution, coated fabrics didn't suffer the loss of its surface optical properties, such as color fading. As seen in Fig. 4e and f, the reflection spectra of the expedited laundered sample was as similar as that of the ordinary one, and the repeated washing just slightly changed the peak position and its intensity. Moreover, the lightness of the coated fabric slightly changed during the whole laundry cycles, and the $K / S$ value of the coated fabric just decreased by about 0.6 compared to that of the original fabric, as shown in Fig. 4g. In other words, if washing is the only reason to degrade its surface property and the laundry frequency is once a week, the coated fabrics in this study could maintain its color for up to at least one year. Thus, the coated fabrics exhibited excellent washability and abrasion durability by the introduction of PA.

Fig. 5 shows the surface morphology of the original NPs/PA coated fabrics, the coated fabrics with 5 times and 10 times of expedite laundering. After 5 cycles and 10 cycles of washing, the NPs could be clearly observed on the coated surface, indicating that the particles were firmly immobilized on the fiber surface, whereas particles on the coated fabrics without the incorporation of PA nearly fell off the fiber surface after just one cycle, as seen in Fig. S6. $\dagger$ In the washing test, PA played an important role in the washing durability for the coated fabrics. The PA solution had a high viscosity, and it appeared at the gap between the particles and fibers or between particles as a firm
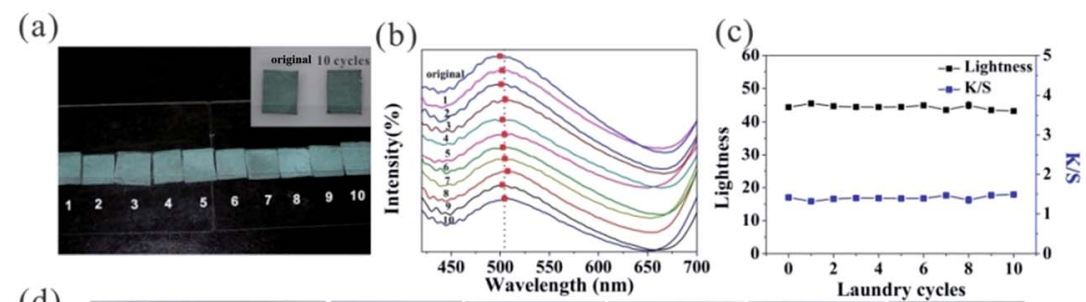

(d)
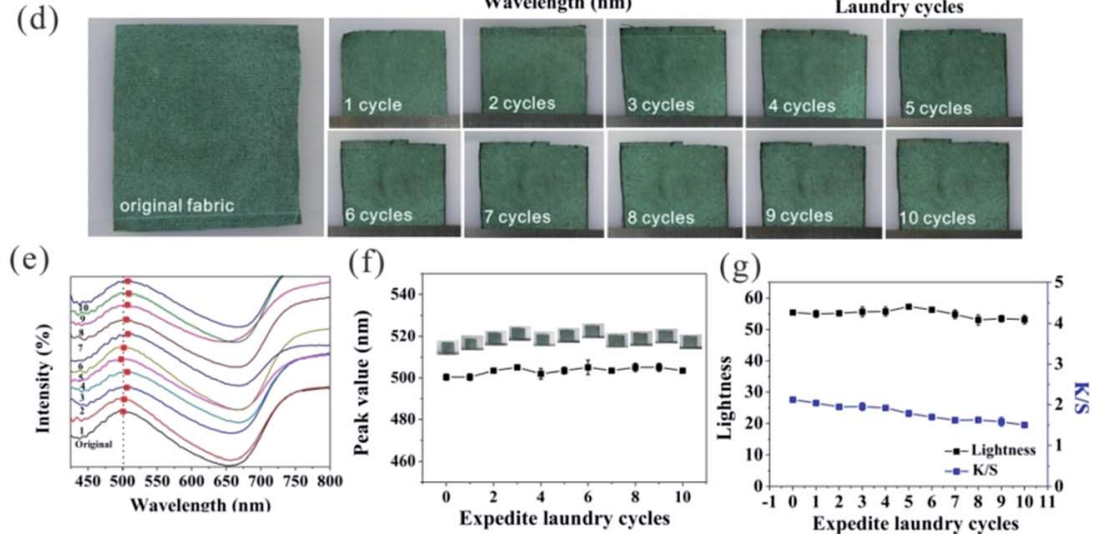

Fig. 4 (a) Images of the coated fabrics with different cycles of standard machine laundry, inset: images of the original fabric and coated fabric with 10 laundry cycles. (b) Reflection spectra of the coated fabric with standard machine laundry from 0 to 10 cycles, and the position of the reflection peak wavelength marked with red dots almost stay in a line under different laundry cycles. (c) Changes in the lightness and $K / S$ value of the coated fabric in (a) with different cycles of standard machine laundry. (d) Images of the coated fabrics with different cycles of expedite machine laundry. (e) Reflection spectra of the coated fabric with expedite machine laundry from 0 to 10 cycles. (f) Plots of the position of the reflection wavelength versus the expedite laundry cycles. (g) Changes in the lightness and K/S value of the coated fabric in (d) with different cycles of expedite machine laundry. 
(a)

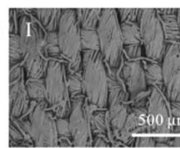

(b)

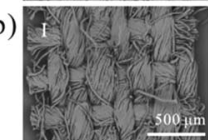

(c)

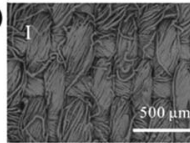

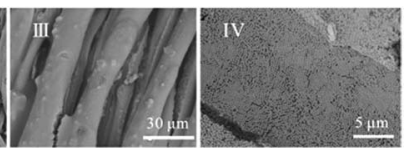
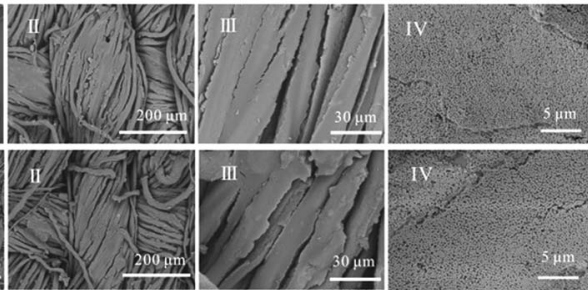

Fig. 5 SEM images of the coated fabrics with different cycles by expedite laundering. (a) 0 cycle, (b) 5 cycles, and (c) 10 cycles. The scale bars are $500 \mu \mathrm{m}, 200 \mu \mathrm{m}, 30 \mu \mathrm{m}$, and $5 \mu \mathrm{m}$ in I, II, III, and IV, respectively.

bridge. Its adhesion strongly depended on the fiber surface roughness, and PA solution covered the rough yarn and tightly bit the fibers, by means of which the particles capped with PA could be fixed on the fabric surface. In addition, $n$-(hydroxymethyl)acrylamide in PA solution had amino group, which would directly react with the hydroxyl groups on the cotton fibers and particle surface respectively at the high temperature of $80{ }^{\circ} \mathrm{C} .{ }^{58} \mathrm{~N}-\mathrm{C}$ and $\mathrm{C}-\mathrm{N}$ bond could be observed by X-ray photoelectron spectroscopy (XPS) and the intensity of the N-C bond in the coated fabrics were more apparent than that in the pure PA solution (see Fig. S7 $\dagger$ ). Thus, the formation of chemical bonds made the fabrics washable to some extent.

For specific application, the wettability of the fabrics is another essential property, such as hydrophobicity. ${ }^{59,60}$ It has been reported that the P(St-MMA-AA) film exhibited the wettability transition by regulating the temperature or $n \mathrm{MMA} / \mathrm{St}$ ratio. ${ }^{61}$ The P(St-MMA-AA) NPs in this study were synthesized in an $n \mathrm{MMA} / \mathrm{St}$ ratio of 0.052 and the water contact angle (CA) of the film was $135^{\circ}$ after $80^{\circ} \mathrm{C}$ thermal treatment. The hydrophobicity and hydrophilicity in the surface chemical component of the colloidal-crystal films was estimated based on the absorbance of the specific IR bands. The characteristic absorbance bands of carboxyl group $\left(1727 \mathrm{~cm}^{-1}\right)$ and benzene group $\left(697 \mathrm{~cm}^{-1}\right.$ ) represented the peak of the hydrophilic and hydrophobic groups, respectively. The absorbance ratio of A697/ A1727 was 6.67, which obviously corresponds to the hydrophobicity (Fig. S8 $\dagger$ ). Higher treating temperatures resulted in improved hydrophobicity. The water CA of the coated film was improved to $150.2^{\circ}$ after $90^{\circ} \mathrm{C}$ treatment. Hydrophobicity of the film showed better improvement tendency as the treatment temperature reached $90{ }^{\circ} \mathrm{C}^{62}$ Surface energy and surface roughness has synergetic effect on the improvement of hydrophobicity of the fabric surface. ${ }^{63}$ We could see from the images obtained using atomic force microscopy (AFM) (Fig. S9†) that the surface of the resulting fiber had a root mean square (RMS) roughness as high as $40 \mathrm{~nm}$ since quasi-amorphous structures created by spray-coating enhanced the surface roughness of the coated fabrics. ${ }^{21}$ Thus, wettability transition on the NPs surface and high surface roughness provided the fabrics with high water repellency. As shown in the inset of the SEM image in Fig.

6a, three drops of $6 \mu \mathrm{l}$ water droplets coloring with different dyes quickly spread and completely wetted the original cotton fabric with an intrinsic contact angle (CA) lower than $5^{\circ}$ (Movie S2, ESI $†$ ), while the same water droplets became spherular on the green coated fabric and the static water CA was $157^{\circ}$ (superhydrophobic) for a long time after treating original fabric by spraying NPs on the surface for 5 times and $80{ }^{\circ} \mathrm{C}$ heating treatment. Original fabric was hydrophilic due to the capillary effect caused by the cotton fibers and the hollow space within the fabric. Water maintained contact with the rough coated structure and did not penetrate the interior texture or the structure of the fabric due to the low surface energy and air trapped at the liquid-solid interface of the fabrics, which is the typical Cassie state. ${ }^{64}$ The whole process was very simple and environmentally friendly, without the introduction of any fluorochemicals. Note that superhydrophobicity of the coating showed excellent stability against washing and abrasion treatment. After 10 cycles of standard machine laundry, the coated fabrics still maintained its superhydrophobicity. The CA underwent a slight decrease with the washing cycles (Fig. S10†). Fig. $6 \mathrm{~b}$ also shows that the coated fabrics could withstand more than 10 laundering cycles with abrasion of the steel balls without a significant reduction in its superhydrophobicity. Apart from the washing and abrasion test, the coating was also very stable under ultrasonic vibrations. After immersing the coated fabric in the beaker filled with water under the condition of $100 \mathrm{~W}$ supersonic vibrations, the coated fabric showed almost no change in its superhydrophobicity (Fig. 6c). The SEM of the ultrasonic vibration treated fabrics confirmed that no morphological change occurred in the coated fiber surface before and after the vibration treatment (Fig. S11 $\dagger$ ). Thus, the great superhydrophobicity and excellent stability of the coated fabrics would effectively prevent the color from fading away when in the wetting environment or under wearing condition.

For coated materials, the exfoliation or physical/chemical damage would directly lead to the loss of surface properties. Partial damage in small domain caused by outer force was expected to be recoverable by facile process in our study. The selfhealing ability of the superhydrophobic coating was investigated by artificially damaging the coated fabric via a vacuum plasma treatment using air as the gas source, which introduced oxygen-containing hydrophilic groups on the coating surface. After $3 \mathrm{~min}$ of plasma treatment, the coated fabric became hydrophilic with a contact angle of $0^{\circ}$ (Fig. S12 $\dagger$ ). However, the original superhydrophobicity of the damaged coating could be restored after exposure at $80^{\circ} \mathrm{C}$ for $20 \mathrm{~min}$, suggesting that the surface of the damaged superhydrophobic coating was again covered with hydrophobic groups. The result was the same as exposing it in the room temperature $\left(25{ }^{\circ} \mathrm{C}\right.$ with a relative humidity (RH) of $55 \%$ ) for $2 \mathrm{~h}$. The coated fabric after plasma treatment was hydrophilic, whereas the surrounding air was hydrophobic. The hydrophobic groups, such as benzene groups, preserved within the coating were expelled and migrated to the coating surface to lower the surface energy. ${ }^{65,66}$ Thus, the oxygen-containing hydrophilic groups or etched coating surface by $\mathrm{O}_{2}$ plasma treatment became buried inside the hydrophilic polyelectrolyte coating. Then, the damaged 
(a)

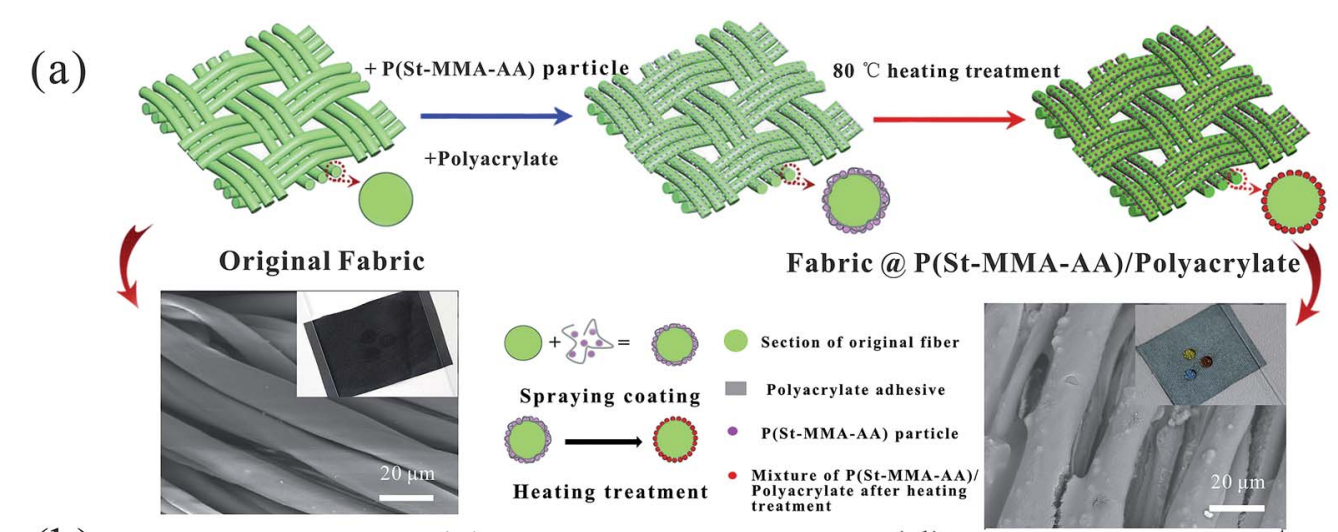

(b)

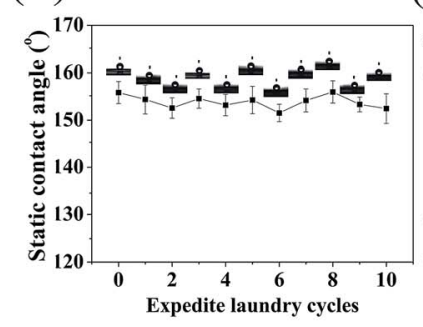

(c)

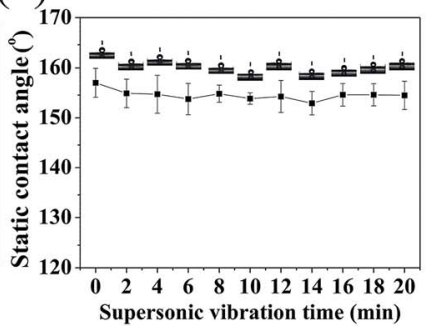

(d)

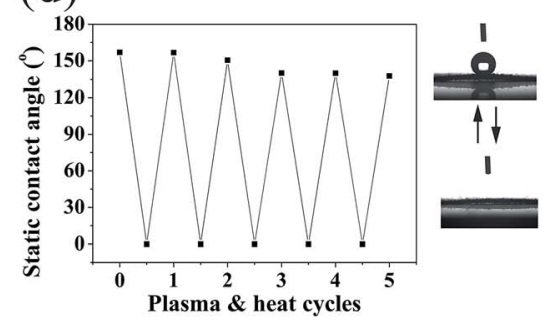

Fig. 6 (a) Coating procedure for superhydrophobic fabrics. (b) CA change with expedite laundry cycles. (c) CA change with supersonic vibration time. (d) Water CA change in the first 5 cycles of plasma-and-heat treatment, and CA of the coated fabric after $\mathrm{O}_{2}$ plasma etching (bottom) and self-healing (top).

fabric was healed. However, the high pressure plasma etching would be unavoidable, breaking down its surface structure. After 4 cycles of etching-healing process, an obvious decline of CA was observed (Fig. 6d) and the original hydrophobic state cannot be recovered by thermal treatment any more. More interestingly, fabrics can be re-sprayed to restore its superhydrophobicity and self-healing ability.

\section{Conclusions}

We prepared robust, washable, self-healing superhydrophobic fabrics with non-iridescent structural color by a one-step spraycoating of the mixed liquor containing P(St-MMA-AA) NPs, PA solution, and $\mathrm{CB}$ nanoparticles to create quasi-amorphous structures with high roughness. The color was non-iridescent and could be tuned by varying the size of the monodispersed $\mathrm{P}$ (St-MMA-AA) NPs. Varying the spraying times made the lightness of the coated fabrics more easily tunable. Introduction of CB was effective to reduce the impact from incoherent multiple scattering of light to enhance the saturability for clearer observations of the structural colors. Incorporation of PA improved the adhesion between the particles and fibers, leading to the improvement of washing and abrasion durability. The high water $\mathrm{CA}\left(>150^{\circ}\right)$ and excellent stability effectively prevented the color from fading away when in the wetting environment. The newly developed spray coating technique is a facile, fast, and low-cost method that is promising for the development of colorful robust self-cleaning clothing, special poster cloth or massive oil spill cleanup, and oil-water separation systems, benefiting the environment and human health. ${ }^{67-69}$

\section{Acknowledgements}

We gratefully acknowledge the financial support received from the National Natural Science Foundation of China under Grant 51073113, 91027039, 51373110 and 11404064, the Natural Science Foundation of the Jiangsu Higher Education Institutions of China under grant 10KJA540046. We also acknowledge support from the (PAPD), Qing Lan Project for Excellent Scientific and Technological Innovation Team of Jiangsu Province (2012), and Project for Jiangsu Scientific and Technological Innovation Team (2013).

\section{Notes and references}

$1 \mathrm{~S}$. Kinoshita and S. Yoshioka, Structural colors in nature: the role of regularity and irregularity in the structure, ChemPhysChem, 2005, 6, 1442-1459.

2 S. Kinoshita, S. Yoshilka and J. Miyazaki, Physics of structural colors, Rep. Prog. Phys., 2008, 71, 076401.

3 T. Yukikazu, Fusion materials for biomimetic structurally colored materials, Polym. J., 2015, 47, 106-113.

4 J. X. Chen, J. Xie, Z. S. Wu, et al., Review of beetle forewing structures and their biomimetic applications in China: (I) on the structural colors and the vertical and horizontal crosssectional structures, Mater. Sci. Eng., C, 2015, 55, 605-619.

$5 \mathrm{~W}$. Yuan, N. Zhou, L. Shi, et al., Structural coloration of colloidal fiber by photonic band gap and resonant mie scattering, ACS Appl. Mater. Interfaces, 2015, 7, 14064-14071.

6 Y. Takeoka, Angle-independent structural coloured amorphous arrays, J. Mater. Chem., 2012, 22, 23299-23309. 
7 R. O. Prum, Structural colouration of mammalian skin: convergent evolution of coherently scattering dermal collagen arrays, J. Exp. Biol., 2004, 207, 2157-2172.

8 E. R. Dufresne, H. Noh, V. Saranathan, et al., Self-assembly of amorphous biophotonic nanostructures by phase separation, Soft Matter, 2009, 5, 1792-1795.

$9 \mathrm{~J} . \mathrm{Zi}, \mathrm{X} . \mathrm{Yu}, \mathrm{Y}$. Li, et al., Coloration strategies in peacock feathers, Proc. Natl. Acad. Sci. U. S. A., 2003, 100, 12576-12578.

10 H. Ryoko, S. Takahiro, K. Kiyofumi, et al., Light-induced saturation change in the angle independent structural coloration of colloidal amorphous arrays, J. Mater. Chem. C, 2014, 2, 344-348.

11 Y. Takeoka, S. Yoshioka, A. Takano, et al., Production of colored pigments with amorphous arrays of black and white colloidal particles, Angew. Chem., Int. Ed. Engl., 2013, 52, 7261-7265.

12 W. H. Chia, D. M. Owen, G. J. Steven, et al., Optimization of sharp and viewing-angle-independent structural color, opt. Express, 2015, 23, 9516.

13 D. Gur, B. Lwshem, M. Pierantoni, et al., Structural Basis for the Brilliant Colors of the Sapphirinid Copepods, J. Am. Chem. Soc., 2015, 137, 8408-8411.

14 Q. Q. Fu, A. Chen, L. Shi, et al., A polycrystalline $\mathrm{SiO}_{2}$ colloidal crystal film with ultra-narrow reflections, Chem. Commun., 2015, 51, 7382-7385.

15 Y. F. Zhang, B. Q. Dong, A. Chen, et al., Using cuttlefish ink as an additive to produce non-iridescent structural colors of high color visibility, Adv. Mater., 2015, 27, 4719-4724.

16 L. Shi, Y. F. Zhang, B. Q. Dong, et al., Amorphous photonic crystals with only short-range order, Adv. Mater., 2013, 25, 5314-5320.

17 H. Yin, B. Dong, X. Liu, et al., Amorphous diamondstructured photonic crystal in the feather barbs of the scarlet macaw, Proc. Natl. Acad. Sci. U. S. A., 2012, 109, 10798-10801.

18 P. Vukusic and J. R. Sambles, Photonic structures in biology, Nature, 2003, 424, 852.

19 A. V. Yu, Z. B. Xiang, C. S. James, et al., On-chip natural assembly of silicon photonic bandgap crystals, Nature, 2001, 414, 289-293.

20 Y. Y. Diao, X. Y. Liu, G. W. Toh, et al., Multiple Structural Coloring of Silk-Fibroin Photonic Crystals and HumidityResponsive Color Sensing, Adv. Funct. Mater., 2013, 23, 5373-5380.

21 D. T. Ge, L. L. Yang, G. X. Wu, et al., Spray coating of superhydrophobic and angle-independent coloured films, Chem. Commun., 2014, 50, 2469-2472.

22 L. Y. Cui, Y. Z. Zhang, J. X. Wang, et al., Ultra-fast fabrication of colloidal photonic crystals by spray coating, Macromol. Rapid Commun., 2009, 30, 598-603.

23 B. Tang, M. Zhang, X. Hou, et al., Coloration of Cotton Fibers with Anisotropic Silver Nanoparticles, Ind. Eng. Chem. Res., 2012, 51, 12807-12813.

24 B. Tang, J. Li, X. Hou, et al., Colorful and Antibacterial Silk Fiber from Anisotropic Silver Nanoparticles, Ind. Eng. Chem. Res., 2013, 52, 4556-4563.
25 B. Tang, J. Wang, S. Xu, et al., Application of anisotropic silver nanoparticles: multifunctionalization of wool fabric original research article, J. Colloid Interface Sci., 2011, 356, 513-518.

26 F. M. Kelly and J. H. Johnston, Colored and Functional Silver Nanoparticle-Wool Fiber Composites, ACS Appl. Mater. Interfaces, 2011, 3, 1083-1092.

27 G. Metraux and C. Mirkin, Rapid Thermal Synthesis of Silver Nanoprisms with Chemically Tailorable, Adv. Mater., 2005, 17, 412-415.

28 Q. Zhang, N. Li, J. Goebl, et al., A Systematic Study of the Synthesis of Silver Nanoplates: Is Citrate a "Magic" Reagent?, J. Am. Chem. Soc., 2011, 133, 18931-18939.

29 D. Aherne, D. Ledwith, M. Gara, et al., Optical Properties and Growth Aspects of Silver Nanoprisms Produced by a Highly Reproducible and Rapid Synthesis at Room Temperature, Adv. Funct. Mater., 2008, 18, 2005-2016.

30 R. R. Arvizo, S. Bhattacharyya, R. A. Kudgus, et al., Intrinsic therapeutic applications of noble metal nanoparticles: past, present and future, Chem. Soc. Rev., 2012, 41, 2943-2970.

31 M. C. Wu, B. H. Ma, T. Z. Pan, et al., Silver-NanoparticleColored Cotton Fabrics with Tunable Colors and Durable Antibacterial and Self-Healing Superhydrophobic Properties, Adv. Funct. Mater., 2016, 26, 569-576.

32 J. Li, L. Yan, Y. Z. Zhao, et al., One-step fabrication of robust fabrics with both-faced superhydrophobicity for the separation and capture of oil from water, Phys. Chem. Chem. Phys., 2015, 17, 6451-6457.

33 P. Spahn, C. E. Finlayson, W. M. Etah, et al., Modification of the refractive-index contrast in polymer opal films, J. Mater. Chem., 2011, 21, 8893-8897.

34 D. T. Ge, E. Lee, L. L. Yang, et al., A robust smart window: reversibly switching from high transparency to angleindependent structural color display, Adv. Mater., 2015, 27, 2489-2495.

35 P. Spahn, C. E. Finlayson, W. M. Etah, et al., Modification of the refractive-index contrast in polymer opal films, J. Mater. Chem., 2011, 21, 8893-8897.

36 R. G. Karunakaran, C. H. Lu, Z. H. Zhang, et al., Highly Transparent Superhydrophobic Surfaces from the Coassembly of Nanoparticles ( $\leq 100 \mathrm{~nm})$, Langmuir, 2011, 27, 4594-4602.

37 L. B. Xu, R. G. Karunakaran, J. Guo, et al., Transparent, Superhydrophobic Surfaces from One-Step Spin Coating of Hydrophobic Nanoparticles, ACS Appl. Mater. Interfaces, 2012, 4, 1118-1125.

38 O. Hitoshi, O. Jun and S. Tetsuo, Facile Fabrication of Colored Superhydrophobic Coatings by Sprayinga Pigment Nanoparticle Suspension, Langmuir, 2011, 27, 9069-9072.

39 G. J. Liu, L. Zhou, C. C. Wang, et al., Study on the high hydrophobicity and its possible mechanism of textile fabric with structural colors of three-dimensional poly(styrene-methacrylic acid) photonic crystals, RSC Adv., 2015, 5, 62855-62863.

40 G. Liu, J. Z. Shao, Y. Zhang, et al., Self-assembly behavior of polystyrene/methacrylic acid (P(St-MAA)) colloidal 
microspheres on polyester fabrics by gravitational sedimentation, J. Text. Inst., 2015, 106, 1293-1305.

41 J. X. Wang, Y. Q. Wen, H. L. Ge, et al., Simple fabrication of full color colloidal crystal films with tough mechanical strength, Macromol. Chem. Phys., 2006, 207, 596-604.

42 G. Liu, L. Zhou, Y. J. Wu, et al., Optical properties of threedimensional P(St-MAA) photonic crystals on polyester fabrics, Opt. Mater., 2015, 42, 72-79.

43 S. H. Li, J. Y. Huang, M. Z. Ge, et al., Robust Flower-Like $\mathrm{TiO}_{2} @$ Cotton Fabrics with Special Wettability for Effective Self-Cleaning and Versatile Oil/Water Separation, $A d v$. Mater. Interfaces, 2015, 2, 1500220.

44 Y. Zhang, G. Q. Zhuang, Y. R. Jia, et al., Structural coloration of polyester fabrics with electrostatic self-assembly of $\left(\mathrm{SiO}_{2}\right)$ PEI $)_{n}$, Text. Res. J., 2016, 1-10.

45 D. T. Ge, L. L. Yang, G. X. Wu, et al., Angle-independent colours from spray coated quasi-amorphous arrays of nanoparticles: combination of constructive interference and Rayleigh scattering, J. Mater. Chem. C, 2014, 2, 43954400.

46 Y. Shiya and T. Yukikazu, Production of colourful pigments consisting of amorphous arrays of silica particles, ChemPhysChem, 2004, 15, 2209-2215.

47 A. R. Parker, Discovery of Functional Iridescence and Its Coevolution with Eyes in the Phylogeny of Ostracoda (Crustacea), Proc. R. Soc. London, Ser. B, 1995, 262, 349-355.

48 P. Vukusic, R. Sambles, C. Lawrence, et al., Sculptedmultilayer optical effects in two species of Papilio butterfly, Appl. Opt., 2000, 40, 1116-1125.

49 Z. Y. Cai, Y. J. Liu, J. H. Teng, et al., Fabrication of Large Domain Crack-Free Colloidal Crystal Heterostructures with Superposition Bandgaps Using Hydrophobic Polystyrene Spheres, ACS Appl. Mater. Interfaces, 2012, 4, 5562-5569.

50 C. Rockstuhl and F. Lederer, Suppression of the local density of states in a medium made of randomly arranged dielectric spheres, Phys. Rev. B: Condens. Matter Mater. Phys., 2009, 79, 132202.

51 S. F. Liew, J. K. Yang, H. Noh, et al., Photonic band gaps in three-dimensional network structures with short-range order, Phys. Rev. A: At., Mol., Opt. Phys., 2011, 84, 063818.

52 R. O. Prum, R. H. Torres, S. Williamson, et al., Coherent light scattering by blue feather barbs, Nature, 1998, 396, 28-29.

53 J. Zi, B. Q. Dong and T. R. Zhan, et al., in Bioinspiration: From Nano to Micro Scales, ed. X. Y. Liu, Springer, New York. 2012, ch. 8.

54 Z. Y. Li, Nanophotonics in China: overviews and highlights, Front. Phys. China., 2012, 7, 601-631.

55 B. Q. Dong, X. H. Liu, T. R. Zhan, et al., Structural coloration and photonic pseudogap in natural random close-packing photonic structures, Opt. Express, 2010, 18, 14430-14438.

56 M. Kohri, Y. Nannichi, T. Taniguchi, et al., Biomimetic noniridescent structural color materials from polydopamine black particles that mimic melanin granules, J. Mater. Chem. C, 2015, 3, 720.

57 M. H. U. Rashid, A. B. Imran, T. Seki, et al., AngleIndependent Structural Color in Colloidal Amorphous Arrays, ChemPhysChem, 2010, 11, 579-583.

58 M. Xiao, Y. W. Li, M. C. Allen, et al., Bio-Inspired Structural Colors Produced via Self-Assembly of Synthetic Melanin Nanoparticles, ACS Nano, 2015, 9, 5454-5460.

59 Y. Meng, B. T. Tang, J. H. Xiu, et al., Simple fabrication of colloidal crystal structural color films with good mechanical stability and high hydrophobicity, Dyes Pigm., 2015, 123, 420-426.

60 Z. G. Xu, Y. Zhao, H. X. Wang, et al., A Super Amphiphobic Coating with an Ammonia-Triggered Transition to Superhydrophilic and Superoleophobic for Oil-Water Separation, Angew. Chem., 2015, 127, 4610-4613.

61 J. X. Wang, Y. Q. Wen, X. J. Feng, et al., Control Over the Wettability of Colloidal Crystal Films by Assembly Temperature, Macromol. Rapid Commun., 2006, 27, 188-192.

62 J. X. Wang, Y. Q. Wen, J. P. Hu, et al., Fine Control of the Wettability Transition Temperature of Colloidal-Crystal Films, Adv. Funct. Mater., 2007, 17, 219-225.

$63 \mathrm{~L} . \mathrm{X} . \mathrm{Li}, \mathrm{B} . \mathrm{C} . \mathrm{Li}, \mathrm{L}$. Fan, et al., Palygorskite@ $\mathrm{Fe}_{3} \mathrm{O}_{4} @$ polyperfluoroalkylsilane nanocomposites for superoleophobic coatings and magnetic liquid marbles, J. Mater. Chem. A, 2016, 4, 5859-5868.

64 J. Y. Huang, S. H. Li, M. Z. Ge, et al., Robust superhydrophobic $\mathrm{TiO}_{2} @$ afabrics for UV shielding, selfcleaning and oil-water separation, J. Mater. Chem. A, 2015, 3, 2825-2832.

65 Y. Li, S. S. Chen, M. C. Wu, et al., All spraying processes for the fabrication of robust, self-healing, superhydrophobic coatings, Adv. Mater., 2014, 26, 3344-3348.

66 H. Zhou, H. Niu, A. Gestos, et al., Robust, Self-Healing Superamphiphobic Fabrics Prepared by Two-Step Coating of Fluoro-Containing Polymer, Fluoroalkyl Silane, and Modified Silica Nanoparticles, Adv. Funct. Mater., 2013, 23, 1664-1670.

67 H. X. Wang, Y. H. Xue, J. Ding, et al., Durable, Self-Healing Superhydrophobic and Superoleophobic Surfaces from Fluorinated-Decyl Polyhedral Oligomeric Silsesquioxane and Hydrolyzed Fluorinated Alkyl Silane, Angew. Chem., Int. Ed., 2011, 50, 11433-11436.

68 S. S. Chen, X. Li, Y. Li, et al., Intumescent Flame-Retardant and Self-Healing Superhydrophobic Coatings on Cotton Fabric, ACS Nano, 2015, 9, 4070-4076.

69 J. Li, L. Yan, X. H. Tang, et al., Robust Superhydrophobic Fabric Bag Filled with Polyurethane Sponges Used for Vacuum-Assisted Continuous and Ultrafast Absorption and Collection of Oils from Water, Adv. Mater. Interfaces, 2016, 3, 1500770-1500778. 